\title{
CUIDADO INTERPROFISSIONAL AO IDOSO COM A DOENÇA DE ALZHEIMER: PERCEPÇÃO DOS FAMILIARES/CUIDADORES
}

\section{Betânia Huppes ${ }^{1}$; Lorena Alves Fiorenza ${ }^{2}$; Francine Casarin ${ }^{3}$; Silomar Ilha ${ }^{4}$}

\section{RESUMO}

O presente estudo possui o objetivo de descrever a importância do cuidado interprofissional com a pessoa idosa com Doença de Alzheimer (DA) na percepção dos cuidadores/familiares. Trata-se de uma etapa da pesquisa-ação de um projeto maior, desenvolvido nas redes de apoio a familiares de pessoas idosas com a DA na cidade de Santa Maria/RS, Brasil. Fizeram parte do estudo seis familiares/cuidadores de pessoas com a DA. Os dados coletados, no mês de abril de 2018, por meio de uma entrevista semiestruturada, sendo submetidos a Análise textual Descritica. A pessoa idosa com DA enfrenta uma série de dificuldades físicas, mentais e sociais. Necessitando de cuidadores/familiares para auxiliar nas atividades de vida diária e o acompanhamento de uma equipe multiprofissional. Os cuidadores relatam a importância do conhecimento/preparo dos profissionais diante deste público, para assim, realizar orientações consisas com a realidade. Reforça-se a importância do cuidado multiprofisisonal/interdisciplinar de qualidade para alcançar resultados positivos.

Palavras-chave: Cuidadores; Doença de Alzheimer; Idoso; Pessoal da Saúde.

Eixo Temático: Atenção Integral e Promoção à Saúde.

\section{INTRODUÇÃO}

A doença de Alzheimer (DA) é um tipo de demência caracterizada por uma neurodegeneração progressiva, manifestada por uma deterioração cognitiva e de

\footnotetext{
1 Acadêmica de enfermagem - UFN. betania.huppes3@gmail.com

2 Acadêmica de enfermagem - UFN. lorenafiorenzza@gmail.com

3 Enfermeira, Mestranda em Ciências da Saúde e da Vida - UFN. fracasarin@ hotmail.com

4 Orientador-Doutor, Professor do Curso de Enfermagem - UFN. silo_sm@ hotmail.com
} 
memória, a qual altera as atividades de vida diária (AVDs), pois as pessoas acometidas pela mesma podem apresentar sintomas neuropsiquiátricos e alterações comportamentais. Essa doença costuma ter início insidioso e se desenvolve de forma lenta e contínua com o passar dos anos. No Brasil, cerca 7,1\% dos idosos acima de 65 anos apresentaram demência e, em 55\% dos casos, a mesma se caracteriza por DA (BRASIL, 2017).

Por se desenvolver, na maioria dos casos, de forma contínua, a DA costuma apresentar três diferentes estágios, sendo eles: leve, moderado e grave. Na forma leve, o indivíduo tem uma perda funcional nas AVDs, mas ainda consegue realizálas. No estágio moderado, a pessoa possui um declínio cognitivo acentuado que a impede de realizar atividades instrumentais e atividades básicas de vida (AIVD). Com a forma grave da doença, o paciente geralmente se torna acamado, apresenta problemas na fala, deglutição e incontinência fecal e urinária, dependendo integralmente de auxílio (MADUREIRA et al., 2018).

Por necessitar de auxílio em suas atividades, a pessoa com DA, geralmente, possui um ou mais cuidadores. O cuidador pode ser formal ou informal, sendo o primeiro uma pessoa que foi treinada para essa função e recebe remuneração para exercer esse trabalho. Já o informal é alguma pessoa do núcleo social que faz esse cuidado de forma voluntária, sem receber pagamento para esse fim. Os cuidadores realizam atividades de higiene oral, corporal, preparo e oferta de alimentos, administração de medicamentos, auxiliam nas eliminações intestinais e urinárias, além de outras tarefas que podem ser requisitadas pela família (ESTADO DE SÃO PAULO, 2014).

Além disso, a pessoa com a DA necessita de um acompanhamento de diversos profissionais, considerando a gravidade da doença neurodegenerativa. Portanto, cuidadores, médicos, enfermeiros, psicólogos, nutricionistas, fisioterapeutas e outros profissionais devem atuar de forma integrada e articulada em prol do melhor cuidado em saúde (MADUREIRA et al., 2018).

É importante, portanto, que a atuação transeda os aspecto profissional ou multiprofissional e contemple a interprofissionalidade em saúde, caracterizada pela noção do trabalho em equipe, utilizando-se da reflexão sobre os papéis 
profissionais, a resolução de problemas e a negociação nos processos de decisão. Esse trabalho deve ser feito com uma construção conjunta de conhecimentos, de forma a respeitar as singularidades dos núcleos de saberes e práticas profissionais (ARAÚJO et al., 2017).

A Atenção Primária em Saúde (APS), por meio da atuação interprofissional possui potencial para auxíliar à pessoa com DA, considerando seu papel de porta de entrada e coordenadora das Redes de Atenção à Saúde (RAS). Os profissional das Estratégias Saúde da Família (ESF) se configuram como importantes atores no cuidado dessas pessoas, considerando que é a ESF a responsável pelo cadastramento dos usuários, pelas visitas domiciliares, pela criação de vínculo usuário/serviço/profissional e pela identificação de fatores que possam necessitar um encaminhamento a outros serviços de saúde (GOYANNA et al., 2017).

A partir disso, o presente estudo possui o objetivo de descrever a importância do cuidado interprofissional com a pessoa idosa com Doença de Alzheimer na percepção dos cuidadores/familiares.

\section{METODOLOGIA}

Trata-se de um estudo, derivado dos dados de um projeto maior, denominado "Cuidado de saúde em rede com vistas à (re)organização familiar de idosos com a doença de Alzheimer na perspectiva da complexidade". Tal projeto trata-se de uma pesquisa-ação, que foi desenvolvida nas redes de apoio a familiares de pessoas idosas com a DA na cidade de Santa Maria/RS, Brasil.

Os dados apresentados nesse estudo, são referentes a etapa inicial da pesquisa-ação, pela identificação das necessidades dentro do contexto. Fizeram parte dessa etapa da pesquisa, familiares/cuidadores de pessoas idosas com a DA, participantes do grupo de apoio Assistência Multidisciplinar Integrada aos Cuidadores de pessoas com a doença de Alzheimer (AMICA), projeto de extensãi desenvolvido na Universidade Franciscana (UFN). Foram considerados como critérios de inclusão: ser familiar/cuidador de uma pessoa idosa com a DA maior de 18 anos; participar do AMICA. 
O convite aos participantes foi realizado pessoalmente em um encontro do AMICA e aqueles que não estavam presentes foram convidados via contato telefônico, disponibilizado aos pesquisadores pela coordenadora do AMICA. Após o aceite, foram agendadas e realizadas, no mês de abril de 2018 , as coletas de dados por meio de uma entrevista semiestruturada contendo a caracterização dos participantes e quatro questões abertas. Salienta-se que algumas entrevistas foram desenvolvidas na universidae dos pesquisadores, outras no domicílio dos participantes, conforme a disponibilidade dos mesmos. Todas foram gravadas em aparelho MP3 e transcritas na íntegra pelos pesquisadores.

Foram convidados um total de 10 familiares/cuidadores que participavam assiduamente dos encontros do AMICA. Atenderam aos critérios de inclusão e aceitaram participar da pesquisa, formando o Corpus desse estudo seis familiares/ cuidadores. Os dados foram analisados com base na técnica de Análise Textual Discursiva, organizada a partir de uma sequência recursiva de três componentes: 1) Unitarização, onde o pesquisador examinou com intensidade e profundidade os textos em detalhes, fragmentando-os no sentido de atingir unidades de significado; 2) estabelecimento de relações, momento em que buscou-se as relações entre as unidades de base, combinandoas e classificando-as, resultando em um ou mais níveis de análise; 3) Comunicação, onde o pesquisador apresentou as compreensões atingidas a partir dos dois focos anteriores, resultando nos metatextos, que foram constituídos de descrição e interpretação dos fenômenos investigados (MORAES; GALIAZZI, 2011).

Foram considerados os preceitos éticos e legais que envolvem a pesquisa com seres humanos, conforme a Resolução número 466/2012 do Conselho Nacional de Saúde (BRASIL, 2012). Os participantes assinaram o Termo de Consentimento Livre e Esclarecido (TCLE), em duas vias, ficando uma com o participante e a outra com o pesquisador. O Projeto foi aprovado pelo Comitê de Ética em Pesquisa da Universidade Franciscana, CAAE: 74984817.3.0000.5306. Para manter o anonimato dos participantes, os mesmos foram identificados pela letra $F$ (Familiar) seguida de um algarismo (F1, F2... F6). 


\section{RESULTADOS E DISCUSSÕES}

Pela singularidade e complexidade dos dados, optou-se por apresenta-los de forma descritiva em um único texto e não fragmentar em diferentes categorias.

A pessoa idosa com DA enfrenta uma série de dificuldades físicas, mentais e sociais, tornando-se necessário a assistência de profissionais da saúde que vão além da identificação fisiopatológica da doença. Os profissionais precisam estar atentos e sensíveis às dificuldades vivenciadas pela pessoa idosa com DA e seus familiares/cuidadores. Deste modo, precisa desenvolver estratégias para preservar a autonomia e qualidade de vida (QV) destes usuários durante as AVDs (ILHA et al, 2016).

Receber atendimento de qualidade é um direito do usuário, desta forma a qualificação dos profissionais vem obtendo avanços ao longo dos anos. Felizmente já está se superando o modelo de prática hospitalocêntrico e fragmentado, buscando-se entender as reais necessidades sociais, contexto social e a epidemiologia local (OLIVEIRA et al, 2016). Portanto, precisa-se de profissionais qualificados para realizar assistência às pessoas idosas com DA e seus cuidadores/familiares. Na fala do cuidador/familiar F6, nota-se a importância do conhecimento para realizar um atendimento de qualidade:

Ah, eu acho muito importante, por que por mais que a pessoa esteja esquecida, mas se tu tratar ela bem, tratar ela com carinho, com amor, as pessoas preparadas para isso, é muito importante, eu sempre digo... não que os outros não saibam, mas os profissionais que entendem da doença de Alzheimer vão ter outro jeito de tratar os pacientes $[\ldots](F 6)$

$\mathrm{Na}$ fala do cuidador/familiar, reforça-se a importância de profissionais preparados para atender pessoas idosas com DA e seus cuidadores/familiares. Um estudo realizado por Aquino et al (2019) com médicos e enfermeiros da ESF, constatou-se que o tema é pouco abordado durante a graduação, sendo muitas vezes focado somente no aspecto fisiopatológico e no diagnóstico, enfatizando pouco estratégias de cuidado ao paciente e seus familiares. Essa situação, contribui 
para o conhecimento insuficiente sobre as abordagens paliativas na assistência de pessoas idosas com DA.

Em outro estudo, realizado com enfermeiros, notou-se a dificuldade dos profissionais em atuar juntamente com os familiares/cuidadores devido a não aceitação da doença. Porém, a família deve ser vista como uma aliada no tratamento deste usuário. Assim, recomenda-se realizar estratégias de acolhimento e suporte para este familiar/cuidador, pois o mesmo está passando por mudanças radicais em seu cotidiano, assim, será possível amenizar sua sobrecarga e oferecer informações importantes para a realização de um cuidado de qualidade (POLTRONIERE; CECCHETTO; SOUZA, 2011). Esse aspecto também é citado na fala do cuidador/familiar F3:

[...] eu acho que a ajuda maior nem é tanto com o idoso. Claro que tem que ter, uma questão de alimentação, remédio e tudo mais, mas eu acho que o cuidador, ele se desgasta muito. Se o cuidador adoecer, quem vai cuidar do idoso? Então é complicado, porque assim desde que a minha mãe foi morar comigo, a minha vida mudou radicalmente. Porque, eu não vou mais nos lugares que eu ia, eu não vou mais jantar à noite, porque eu sei que eu vou demorar pra voltar [...] (F3).

Desta forma nota-se a importância da criação de capacitações/palestras/cursos de atualizações, para ampliar o conhecimento dos profissionais da saúde, principalmente acerca da realidade/contexto da pessoa idosa com DA e seus familiares/cuidadores. A Política Nacional de Educação Permanente poderá enfatizar os cuidados, gerontotecnologias e alternativas terapêuticas diante da DA em suas atividades educativas. Assim, os profissionais também poderão instruir os cuidadores/familiares, oferecendo o suporte necessário para ambos (BRASIL, 2013).

Todavia, outra dificuldade relatada pelos profissionais da saúde, é a falta de uma equipe ampliada na atenção primária, como: fisioterapeutas, nutricionistas, fonoaudiólogos, psicólogos, entre outros, para promover a qualidade de vida aos pacientes, sendo possível reduzir encaminhamentos para centros de serviços especializados e oferecer mais atendimentos mensais aos usuários (EQUINO et al, 
2019). Alguns entrevistados frisaram a importância do acompanhamento profissional adequado da pessoa com DA:

[...] e uma fonoaudióloga, que por sinal foi uma evolução, não digo uma evolução, mas foi uma coisa muito interessante... a minha mãe tava se afogando com a alimentação, principalmente, se ia comer um arroz ou um pão, alguma coisa sequinha assim, né...ela tava se afogando, né...aí veio o trabalho da fonoaudióloga fazer exercícios para fortalecer a musculatura superior [...] (F2)

$\mathrm{Na}$ fala do F2 pode-se evidenciar a relevância do acompanhamento pelo profissional fonoaudiólogo. A pessoa com a DA em seu estágio mais avançado, comumente apresenta dificuldades para manipular e mastigar o bolo alimentar, além de não conseguir deglutir. Estudos mostram que a disfagia é evidenciada em muitos pacientes com DA, sendo considerada uma das principais causas de pneumonia aspirativa. Dessa maneira, o profissional fonoaudiólogo, além de identificar as dificuldades dos pacientes, possui o dever de informar e educar a família e cuidadores quanto a melhor maneira de alimentar o indivíduo (PEREZ-RAMOS et al., 2016).

Além do fonoaudiólogo, o profissional nutricionista, odontólogo e enfermeiro também são citados nas falas dos entrevistados.

[...] toda a ajuda é bem-vinda. Não tem como tu escolher uma nutrição. Porque às vezes a gente tenta fazer o melhor mas às vezes não é o suficiente. E às vezes a gente acha que é melhor e tá errando, pecando em algum lugar. (F1)

Recebi orientação sobre a alimentação, que tem que dar uma comida mais pastosa, dependendo do grau que o paciente se encontra, alimentação sobre frutas, verduras, pra ter uma qualidade de vida melhor, o sono, o pessoal da Nutrição. Já tivemos com a Odonto, pessoal da Odonto, teve a professor de Enfermagem que fez sobre a medicação, então a gente tem orientação. Dá bastante apoio nesse sentido

A nutrição é de suma importância para os pacientes com DA, pois uma nutrição inadequada pode favorecer a progressão rápida da doença, assim como a nutrição adequada pode retardar esse processo. Além disso, esse profissional é 
habilitado para mensurar quais as necessidades nutricionais de cada indivíduo. $O$ nutricionista atua em conjunto com o fonoaudiólogo e os dois possuem o dever de orientar a família acerca da nutrição adequada dos pacientes a fim de reduzir possíveis danos que a DA possa trazer (PEREIRA et al., 2019).

Devido ao comprometimento funcional e cognitivo causado pela DA, os pacientes apresentam problemas em relação à saúde bucal, sendo periodontite e a cárie dentária umas das principais delas. Dessa maneira, o acompanhamento realizado pelo dentista é essencial para a qualidade de vida do indivíduo. Um estudo feito por Telles, Silva e Vidal (2021), evidenciou a existência da relação entre periodontite e a DA. Portanto, o cirurgião dentista pode ser um dos primeiros a verificar um possível diagnóstico de demência, devendo comunicar a família e equipe de saúde para a realização de exames e procedimentos necessários.

O enfermeiro é o profissional responsável pela realização das ações de educação e promoção de saúde, no intuito de fortalecer a autonomia dos usuários e comunidade. Ainda, quando inserido na APS, possui o dever de avaliar o território, identificando as demandas mais prevalentes no local. Em casos de pacientes com DA, o enfermeiro deve realizar visitas domiciliares em conjunto com os ACS, orientar familiares e cuidadores acerca do cuidado à saúde, além de encaminhar o usuário a outros serviços da RAS, em casos que se necessite da intervenção de outros profissionais que não trabalham na ESF do território (GUIMARÃES, 2018).

Apesar de estudos sobre as ações multidisciplinares e interdisciplinares ainda serem escassas em relação a pessoa com DA, elas podem oferecer cuidados mais efetivos para a pessoa idosa e seus familiares/cuidadores. Assim, compreende-se a que seja possível maximizar seus níveis cognitivos e funcionais, bem como diminuir os custos e proporcionar maior QV ao paciente e familiares (BERTAZONE et al, 2016).

\section{CONCLUSÃO}

O presente estudo reforçou a importância da equipe multiprofissional, do trabalho interprofissional e de profissionais capacitados para realizar a assistência de qualidade a pessoas idosas com DA, na percepção dos próprios 
cuidadores/familiares. A equipe interprofissional deve estar inserida em todos os contextos, sendo uma forte aliada tanto no tratamento quanto na realização da educação em saúde com os familiares e/ou cuidadores.

A vista disso, também enfatiza-se a importância de abordar assuntos relacionados a DA na graduação dos cursos da área da saúde, considerando a relevância do conhecimento prático e científico, além do domínio fisiopatológico para o diagnóstico precoce, o tratamento e para a melhoria da QV da pessoa com DA. Também se destaca a importância das atividades de educação em saúde em relação a DA com os acadêmicos e profissionais, obtendo pessoas preparadas para amenizar os desafios que a doença provoca a pessoa idosa e também realizar o devido acolhimento e assistência aos familiares/cuidadores.

\section{REFERÊNCIAS}

AQUINO, E. V. D. O. et al. Conhecimentos dos Profissionais na Atenção Básica sobre Cuidados Paliativos para Doença De Alzheimer. Trabalho de Curso apresentado à disciplina de Iniciação Científica do Curso Medicina do Centro Universitário UniEVANGÉLICA, Anápolis - Goiás, p. 1-48, jan./2019. Disponível em:

http://repositorio.aee.edu.br/bitstream/aee/1367/1/TCC\%20FINAL\%20ALZHEIMER\% 20corrigido.pdf. Acesso em: 31 ago. 2021.

ARAÚJO, T. A. M. D. et al. Multiprofissionalidade e interprofissionalidade em uma residência hospitalar: o olhar de residentes e preceptores. Interface, Botucatu, v. 21, n. 62, p. 601-613, jul./2017. Disponível em: https://www.scielo.br/j/icse/a/XNR9GMyVnXx6v85LVPk3kLy/?lang=pt\&format=pdf. Acesso em: 31 ago. 2021.

BERTAZONE, T. M. A. et al. Ações multidisciplinares/interdisciplinares no cuidado ao idoso com Doença de Alzheimer. Rev Rene, Fortaleza, v. 17, n. 1, p. 144-153, fev./2016. Disponível em: http://periodicos.ufc.br/rene/article/view/2633/2020. Acesso em: 31 ago. 2021. 
BRASIL. Ministério da Saúde. Secretaria de Atenção à Saúde. Portaria Conjunta № 13, de 28 de novembro de 2017. Aprova o Protocolo Clínico e Diretrizes Terapêuticas da Doença de Alzheimer. Diário Oficial da União. 28 nov. 2017.

BRASIL, Política Nacional de Educação Permanente do SUAS/ Ministério do Desenvolvimento Social e Combate à Fome. $1^{\text {a }}$ ed. Brasília: MDS, 2013, 57p.

GOVERNO DO ESTADO DE SÃO PAULO. Instituto de Assistência Médica ao Servidor Público Estadual (lamspe). Manual para Cuidadores de Idosos. Iamspe: São Paulo. fev. 2014.

GOYANNA, N. F. et al. Idosos com doença de alzheimer: como vivem e percebem a atenção na estratégia saúde da família. Revista de Pesquisa Cuidado É Fundamental Online, Rio de Janeiro, v. 9, n. 2, p. 379-386, abr./2017. Disponível em: http://www.seer.unirio.br/cuidadofundamental/article/view/5037/pdf_1. Acesso em: 30 ago. 2021.

GUIMARÃES, M. H. D. Doença de Alzheimer: Papel do Enfermeiro como Promotor de Saúde. Revista Científica Multidisciplinar Núcleo do Conhecimento, São Paulo, v. 06, n. 03, p. 78-88, jun./2018. Disponível em: https://www.nucleodoconhecimento.com.br/saude/doenca-de-alzheimer. Acesso em: 1 set. 2021.

ILHA, S. et al. Doença de alzheimer na pessoa idosa/família: Dificuldades vivenciadas e estratégias de cuidado. Escola Anna Nery [online], Rio de Janeiro, v. 20, n. 1, p. 138-146, mar./2016. Disponível em: https://www.scielo.br/j/ean/a/JfKX6jZsVXSWCpKYQHm8Wzj/?format=pdf\&lang=pt. Acesso em: 30 ago. 2021.

MADUREIRA, B. G. et al. Efeitos de programas de reabilitação multidisciplinar no tratamento de pacientes com doença de Alzheimer: uma revisão sistemática.. Cadernos Saúde Coletiva, Rio de Janeiro, v. 26, n. 2, p. 222-232, jun./2018. Disponível em: 
https://www.scielo.br/j/cadsc/a/f5HGg8NjBHMxZ3njY9dTZnJ/?lang=pt\&format=pdf. Acesso em: 30 ago. 2021.

MORAES, Roque; GALIAZZI, Maria Carmo. Análise textual discursiva. 3. ed. Ijuí: Unijuí, 2011. p. 224-224.

OLIVEIRA, M. P. R. D. et al. Formação e Qualificação de Profissionais de Saúde: Fatores Associados à Qualidade da Atenção Primária. Revista Brasileira de Educação Médica, Brasília, v. 40, n. 4, p. 547-559, mar./2016. Disponível em: https://www.scielo.br/j/rbem/a/9xmh853N9RkL7F8x97XFxxh/?format=pdf. Acesso em: 1 set. 2021.

PEREIRA, M. M. L. et al. Atuação do Nutricionista no tratamento de paciente com Doença de Alzheimer: relato de caso. Revista Thema, Pelotas, v. 16, n. 3, p. 531536 , out./2019.

Disponível

em: https://periodicos.ifsul.edu.br/index.php/thema/article/view/1351/1254. Acesso em: 29 ago. 2021.

PEREZ-RAMOS, I. C. S. et al. Percepção dos Cuidadores sobre as Alterações de Deglutição Causadas pela Demência. Revista Brasileira de Ciências da Saúde, Paraíba, v. 20, n. 2, p. 127-132, jun./2016. Disponível em: https://periodicos.ufpb.br/ojs2/index.php/rbcs/article/view/24552. Acesso em: 30 ago. 2021.

POLTRONIEREA, Silvana; CECCHETTO, Fátima Helena; SOUZA, E. N. D. Doença de Alzheimer e demandas de cuidados: o que os enfermeiros sabem?. Revista Gaúcha de Enfermagem [online], Porto Alegre, v. 32, n. 2, p. 270-278, 2011. , Porto Alegre, v. 32, n. 2, p. 270-278, jun./2011. Disponível em: https://www.scielo.br/j/rgenf/a/3cYxYjqCSTd7dBDmT8P58cJ/?format=pdf\&lang=pt. Acesso em: 1 set. 2021.

TELLES, Ingrid Paula; SILVA, J. D. M. S; VIDAL, Priscila Pavan. Associação entre a doença periodontal e a doença de Alzheimer e a importância da atuação do 


\section{QUFN}

cirurgião-dentista no paciente portador dessas condições. Ciência Atual: Revista Multidisciplinar da UniSãoJosé, Rio de Janeiro, v. 15, n. 1, p. 36-46, jan./2021. Disponível em: https://revista.saojose.br/index.php/cafsj/article/view/397. Acesso em: 1 set. 2021. 